\title{
Neuropsychological Assessment of Preschoolers
}

\author{
Ida Sue Baron • Peter John Anderson
}

Received: 16 October 2012 / Accepted: 22 October 2012 /Published online: 30 October 2012

(C) Springer Science+Business Media New York 2012

The assessment of preschoolers (children aged 3-6 years) has been an understudied area of child neuropsychology practice that is deserving of an intensified focus across child health disciplines. A number of limitations have contributed to a regrettable situation in which the years between infancy and school entry have been given sparse attention and limited resources. Prominent among these has been the relative absence of standardized developmentally suitable neuropsychological instrumentation with appropriate psychometric properties and normative data for very young children. An additional limitation has been the substantial gap in graduate level teaching and subsequent resident training related to preschool developmental issues, as well as effective techniques that enhance valid evaluation of infants and young children. This has left many young professionals without a strong didactic or experiential basis. Lacking the adequate expertise and skills necessary for the unique challenge of validly testing very young children, many have restricted their clinical practice to children who are of at least school age.

The confluence of factors that has contributed to a pattern of infrequent referral of preschoolers for clinical

\section{S. Baron $(\bowtie)$}

Independent Private Practice,

10116 Weatherwood Court,

Potomac, MD 20854, USA

e-mail: ida@isbaron.com

\section{P. J. Anderson}

Murdoch Research Institute,

Melbourne, Australia

\section{S. Baron}

Departments of Neurosciences and Pediatrics,

Inova Children's Hospital,

Falls Church, VA, USA

P. J. Anderson

University of Melbourne,

Melbourne, Australia neuropsychological evaluation relative to the number of evaluations requested for older children has also resulted in an inaccurate perception among referral sources that a neuropsychological assessment is not useful before a child reaches school age, when deficits are most likely to become explicitly apparent. However, the standard reliance on a test of general intelligence and periodic tracking of motor, language, and social developmental milestones are insufficient coverage of brain-behavioral functioning in these early years. Indeed, adverse effects on brain development can be documented during this formative maturational period. These crucial early years have been under-examined and insufficiently researched even though pediatric neuropsychologists are especially well-suited to identify emergent delay or dysfunction in the years before school entry. Further, the omission of such early examination has had the untoward effect of delaying pragmatic advances in early-age identification of neuropsychological delay and disorder. Such delay has restrained opportunities for innovative early intervention and treatment that might offset deficits manifesting more obviously during the school years. We would argue that the preschool years are an ideal age to intervene given the dramatic brain growth and cognitive development that occurs during this period.

Thus, the aim of this Special Issue was two-fold: 1) to assemble experts in early assessment of specific cognitive domains to bring greater attention to the critically important but less well understood preschool years, and 2) to provide the reader with reviews that elucidate the value of neuropsychological assessment in this early developmental period. While understanding of the course of brain development in typically developing children and its associated progressive and regressive processes has expanded dramatically as remarkable technological advances enable improved visualization of brain structure and function, assessment of preschoolers has not kept pace. 
This Special Issue begins with Brown \& Jernigan's rare coverage of brain development and maturation focused specifically on the formative preschool years. They elegantly review the scientific tools that are broadening understanding of structural and functional brain development through the preschool years. Their coverage of the neurobiological foundations of early postnatal development and physiological brain development while emphasizing methodological factors has resulted in a seminal contribution that is essential reading and provides a solid foundation for the domain articles that follow.

The authors of each subsequent paper provide an informative and expanded context for understanding methods that have been specifically employed to enable measurement with their specific areas of interest. Included are reviews of assessment in preschoolers within the neuropsychological domains of intelligence, executive function, attention, language, motor, and memory function. Each domain paper includes discussion of the neurological basis of the respective function under review, as well as a range of psychological methods that either support reliable and valid clinical assessment or have been shown to have experimental usefulness. Together, the papers of this Special Issue emphasize a crucial point: that accurate assessment at a very young age will provide meaningful data that enhances early identification of delay or deficit and, in turn, will provide an evidence-based rationale for early intervention.

Baron and Leonberger review relevant developments and theoretical positions that have been central in the history of intelligence testing. This discussion includes the advantages of intelligence testing as well as cautions regarding the limitations inherent when using multidimensional tests. The authors emphasize that while intelligence tests are not neuropsychological measures, they are useful as a means to generate hypotheses about brain-behavior functioning that may be subsequently addressed through a comprehensive neuropsychological evaluation that includes consideration of the individual's personal history and clinical context.

Anderson and Reidy's paper on executive function assessment provides support for early age measurement of diverse executive capacities to provide timely application of interventional strategies and informed understanding of the varieties of delay or dysfunction that may be detected as executive skills mature. They summarize the Executive Control System model of executive function, which provides a context for their review of the neural systems that underlie executive skill development, discuss experimental paradigms that have proven useful and the diverse clinical measures available to pediatric neuropsychologists who wish to assess executive function components in the preschool years, and describe the enormous maturation in executive functioning that occurs in this developmental period.

Mahone and Schneider's review of assessment of attention and attention deficit/hyperactivity disorder (ADHD) includes a summary of early developmental risk factors and of neuropsychological models of attention as they relate to brain development over the preschool years. The authors identify behavioral and pharmacological interventions that have demonstrated applicability and present a detailed review of available performance-based measures, structured caregiver interviews, and third party rating scales that have been enlisted to assess attention and ADHD in preschoolers.

Conti-Ramsden and Durkin review the challenges related to the assessment of normal language milestone accomplishment and specific language impairment in preschoolers, for whom language acquisition is highly variable. They identify key language developmental milestones, noting how differences in timing experienced by young children complicate formal assessment. They further examine the neurobiological bases for successful language acquisition and impairment of this critical human ability. A more complex assessment than is typically considered is endorsed, and the means to accomplish the necessary wider exploration of the nature of language skill acquisition in this early developmental period is suggested, while the limitations inherent to these existent measures are acknowledged.

Assessment of motor function, reviewed by Piek, Hands and Licari, includes a discussion of maturational changes occurring during brain development at the preschool level and of sex differences and their influence on the development of motor ability. A discussion of available assessment instruments that assess either overall motor performance or that are useful to assess specific performance deficits is included, along with intervention approaches. As well, a relevant discussion about how to discriminate delay from impairment at such a young age and in context with comorbid disorder is an important contribution to the literature on young age assessment.

In a review of memory assessment at preschool age, Bauer, Leventon and Varga address the substantial and often unresolved complexities associated with attempts to validly measure learning and retrieval in those whose young age raises natural obstacles to psychological assessment in general, and to emergent memory measurement in particular. The neural bases of memory stages are summarized and examples of standardized and experimental episodic memory measures are described, along with helpful insights about the benefits as well as limitations of both individual instruments and memory batteries that have gained wide acceptance in the pediatric neuropsychologist's armamentarium.

Our hope is that this Special Issue will stimulate further discussion about the value of early detection and remediation of neuropsychological deficits. We acknowledge that test developers and publishers have devoted effort and resources in developing preschool measures for assessing general intelligence, language, and motor development. Despite those efforts, other cognitive domains have been neglected and will continue to be until those in the field demand access to standardized measures. 